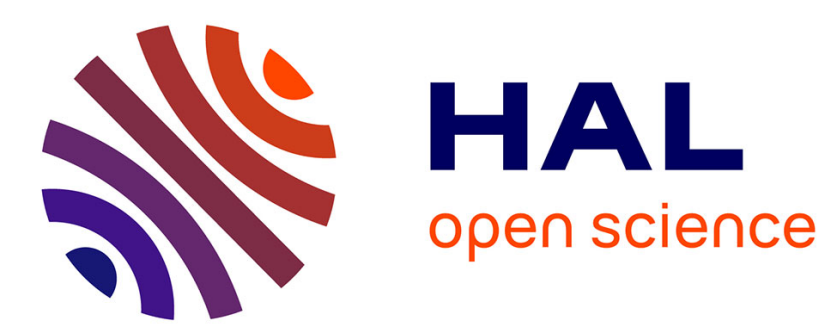

\title{
Terahertz adaptive optics with a deformable mirror
}

Mathilde Brossard, Jean-François Sauvage, Mathias Perrin, Emmanuel

Abraham

\section{To cite this version:}

Mathilde Brossard, Jean-François Sauvage, Mathias Perrin, Emmanuel Abraham. Terahertz adaptive optics with a deformable mirror. Optics Letters, 2018, 43 (7), pp.1594-1597. 10.1364/OL.43.001594 . hal-03525133

\section{HAL Id: hal-03525133 \\ https://hal.science/hal-03525133}

Submitted on 13 Jan 2022

HAL is a multi-disciplinary open access archive for the deposit and dissemination of scientific research documents, whether they are published or not. The documents may come from teaching and research institutions in France or abroad, or from public or private research centers.
L'archive ouverte pluridisciplinaire HAL, est destinée au dépôt et à la diffusion de documents scientifiques de niveau recherche, publiés ou non, émanant des établissements d'enseignement et de recherche français ou étrangers, des laboratoires publics ou privés. 


\title{
Terahertz adaptive optics with a deformable mirror
}

\author{
Mathilde Brossard ${ }^{1,2}$, Jean-Françols Sauvage ${ }^{3,4}$, \\ Mathias PerRin ${ }^{1}$, and Emmanuelabraham ${ }^{1, *}$ \\ ${ }^{1}$ Univ. Bordeaux, CNRS, LOMA, UMR 5798, F-33400 Talence, France \\ ${ }^{2}$ NeTHIS - New Terahertz Imaging Systems, F-33700 Mérignac, France \\ ${ }^{3}$ Onera - The French Aerospace Lab, F-92322 Châtillon, France \\ ${ }^{4}$ Aix-Marseille Université, CNRS, LAM, UMR 7326, F-13013, France \\ *Corresponding author: emmanuel.abraham@u-bordeaux.fr
}

September 18, 2019

\begin{abstract}
We report on the wavefront correction of a terahertz beam using adaptive optics which requires a wavefront sensor able to sense the optical aberrations and a wavefront corrector. The wavefront sensor relies on a direct 2D electro-optic imaging system composed of a $\mathrm{ZnTe}$ crystal and a CMOS camera. By measuring the phase variation of the terahertz electric field in the crystal, we were able to minimize the geometrical aberrations of the beam thanks to the action of a deformable mirror. This phase control will open the route to terahertz adaptive optics in order to optimize the $\mathrm{THz}$ beam quality for both practical and fundamental applications. (๑) 2019 Optical Society of America
\end{abstract}

OCIS codes: ciscodes

http://dx.doi.org/10.1364/ao.XX.XXXXXX

Wavefront control of optical beams is essential to lessen the geometrical aberrations and optimize the performances of an optical system. This aspect is essential in astronomy where the turbulence of the atmosphere strongly affects the electromagnetic waves detected by the telescopes in visible and near-infrared spectral regions. The dynamic evolution of the atmosphere induces strong changes in the air optical index which strongly corrugates the image quality of astronomical imagers [1]. This problem has been efficiently solved with the emergence of adaptive optics $(\mathrm{AO})$ which combines the use of a wavefront analyzer together with a deformable mirror (DM) which can correct in realtime the turbulence of the atmosphere. At present, for visible and near-infrared wavelengths, commercially available AO systems can control the geometrical aberrations of an optical system, providing a mature technology for many applications including not only astronomy, but also ophthalmology and microscopy [2]. In practice, wavefront meaurement can be accomplished with a Shack-Hartmann sensor that can locally measure the wavefront slopes of an incoming optical beam [3]. A computer allows to estimate the wavefront shape from the slope measurements and deduce the shape to be compensated by the DM. Various DM technological concepts have been developed to achieve the wavefront control and provide the final correction of the optical aberrations. These specifically designed mirrors are characterized by the number of actuators used to deform the mirror surface, the actuator pitch and stroke, and its response time to the external control signal. Typically, for the first systems ded- icated to astronomy, 180 actuators in total where requested to provide an efficient correction in the infrared. In the last systems installed at VLT [4], up to 1300 actuators in total are required for a high level correction in the visible spectrum.

In the terahertz (THz) spectral domain, it is still challenging to correct the spatial profile and wavefront of a beam. The wavefront measurement in itself is not straightforward, due to the lack of effective $\mathrm{THz} 2 \mathrm{D}$ detectors. However, this measurement is essential to optimize the spatial resolution or illumination uniformity of THz imaging systems, developed for a large variety of applications such as security and defense, non-destructive testing of industrial materials, etc. [5]. Wavefront measurement of $\mathrm{THz}$ pulses is also mandatory for the control of far-field intensity distribution of time-domain spectrometers or to increase the peak power of intense terahertz sources and optimize the beam focalization of scanning $\mathrm{THz}$ time-domain imaging systems [6].

The measurement of $\mathrm{THz}$ wavefronts for both continuous wave and pulsed sources has been tentatively studied in the past few years. In 2008, Bitzer et al. determined the beam profiles of $\mathrm{THz}$ pulses after passing through a hyper-hemispherical silicon lens [7]. In 2012 and 2013, Cui et al. proposed the first $\mathrm{THz}$ wavefront measurement associated with a Hartmann sensor and indirect scanning method, detecting the radiation with a pyroelectric sensor $[8,9]$. Richter et al. presented a similar arrangement with a 2D detection consisting in a microbolometer camera $[10,11]$. Another aperture scanning device was proposed to characterize the $\mathrm{THz}$ beam propagation in a time-domain spectrometer [12]. In 2016, we proposed the wavefront characterization of $\mathrm{THz}$ pulses using a Hartmann sensor associated with a 2D electro-optic (EO) imaging system [13]. Nevertheless, our indirect method used a metallic mask which limited the beam transmission and required an initial calibration or reference measurement. More recently, we reported on the development of a wavefront sensor for $\mathrm{THz}$ pulses using a direct 2D EO imaging system composed of a ZnTe crystal and a CMOS camera [14]. We were able to reconstruct the $\mathrm{THz}$ wavefront in order to determine the geometrical aberrations of the optical system without any prior reference measurement.

In this letter, we propose to combine this direct wavefront measurement of $\mathrm{THz}$ pulses with the simultaneous utilization of a DM in order to minimize the geometrical aberrations of the $\mathrm{THz}$ beam. The device is adapted for $\mathrm{THz}$ sources delivering short and intense $\mathrm{THz}$ pulses such as air plasma $\mathrm{THz}$ generation and optical rectification in nonlinear crystals. After verifying that the system is able to properly measure and control the $\mathrm{THz}$ wavefront modified by the action of the DM, we will finally 
make the demonstration of an adaptive correction of geometrical aberrations on a $\mathrm{THz}$ beam.

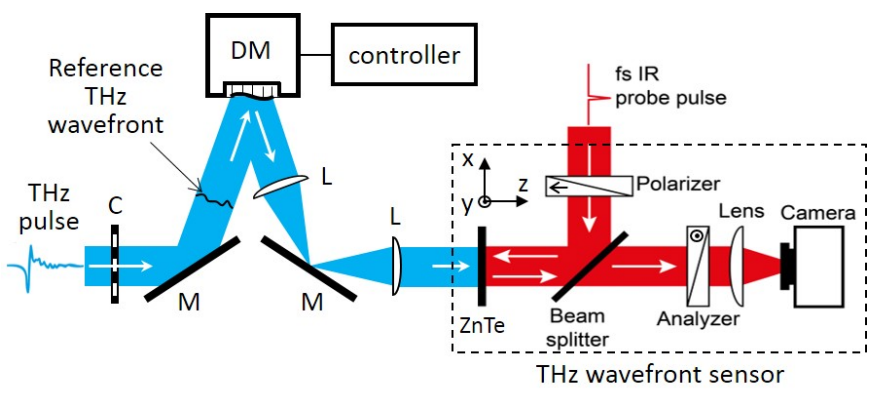

Fig. 1. Experimental setup. DM: ALPAO deformable mirror, $\mathrm{C}$ : chopper, M: metallic planar mirror, L: plano-convex $\mathrm{THz}$ lens, $f^{\prime}=50 \mathrm{~mm}$ focal length.

Broadband $\mathrm{THz}$ pulses $(0.1-3 \mathrm{THz})$ were generated by optical rectification of amplified femtosecond laser pulses $(800 \mathrm{~nm}, 1 \mathrm{~mJ}$, $150 \mathrm{fs}, 1 \mathrm{kHz}$ repetition rate) in a $1 \mathrm{~mm}$-thick ZnTe crystal with a clear aperture of $25 \mathrm{~mm}$. In order to measure and correct the wavefront of the $\mathrm{THz}$ electric field, the beam is sent on the $\mathrm{DM}$ at $\alpha=16^{\circ}$ incidence angle with respect to the normal of the mirror (Fig. 1). This latter is commercialized by ALPAO, specialized in AO components and systems. The DM 97-15 model has the following characteristics: aluminium membrane, 97 magnetic actuators, $13.5 \mathrm{~mm}$ pupil diameter, $1.5 \mathrm{~mm}$ pitch and 11 actuators across a diameter. The tip/tilt stroke is $>60 \mu \mathrm{m}$ $\mathrm{PV}$ and the defocus/astigmatism stroke is $>40 \mu \mathrm{m}$ PV. The local deformation between two adjacent actuators is typically $>5 \mu \mathrm{m}$ and the setting time is as fast as $800 \mu \mathrm{s}$. It is important to note that this mirror is not perfectly adapted to $\mathrm{THz}$ frequencies since the $5 \mu \mathrm{m}$ total course of each actuator is 60 times smaller than the wavelength at $1 \mathrm{THz}$. Therefore, we have to bear in mind that this mirror will not be able to induce large optical phase shifts and correct large geometrical aberrations in the $\mathrm{THz}$ spectral range.

After the reflection on the DM, the $\mathrm{THz}$ beam is sent into a second $1 \mathrm{~mm}$-thick ZnTe crystal for the detection of the THz electric field. Additionaly, we used two plano-convex $\mathrm{THz}$ lenses with $f^{\prime}=50 \mathrm{~mm}$ focal lengths to conjugate the deformable mirror plane with the ZnTe crystal one in a classical $4 f^{\prime}$ configuration with a 1:1 magnification. In this condition, the phase shift induced by the deformable mirror on the $\mathrm{THz}$ wave is directly transferred to the crystal plane where the wavefront is measured. Thanks to a collinear EO detection with a time-delayed femtosecond laser probe pulse reflected by the front (left side in Fig. 1) face of the crystal, we are able to measure the $\mathrm{THz}$ electric field by capturing the laser probe intensity with a CMOS camera [14]. Thanks to the objective lens located in front of the camera, the crystal plane is conjugated with the CMOS chip. Consequently, the DM is actually conjugated with the camera. For a given time delay $t$ between the $\mathrm{THz}$ and the probe pulses, the system is able to provide a $2 \mathrm{D}$ ( $\mathrm{x}$-horizontal and $\mathrm{y}$-vertical directions) image at $800 \mathrm{~nm}$ corresponding to the distribution of the THz electric field $E_{T H z}(x, y, t)$ at the crystal position. This image can be obtained with a $500 \mathrm{~Hz}$ acquisition rate thanks to the synchronization with the laser repetition rate and a dynamic subtraction method [15]. The experimental procedure to measure the $\mathrm{THz}$ wavefront has been detailed in [14]. Shortly, for every pixels of the camera, i.e. for every $x$ and $y$ positions in the ZnTe crystal plane, it is possible to plot the corresponding temporal waveform $E_{T H z}(t)$. Then, through a Fourier transformation of the temporal data, it is possible to calculate the amplitude and the phase of the complex electric field $E_{T H z}(\Omega)$, where $\Omega$ is the THz frequency. In our experiment, the $\mathrm{THz}$ spectrum extends from 0 to $3 \mathrm{THz}$ [14]. It is next straightforward to plot the amplitude and the relative phase of $E_{T H z}(x, y, \Omega)$ and obtain the final wavefront from the phase map, characterized by the surface of equiphase of the electromagnetic field.
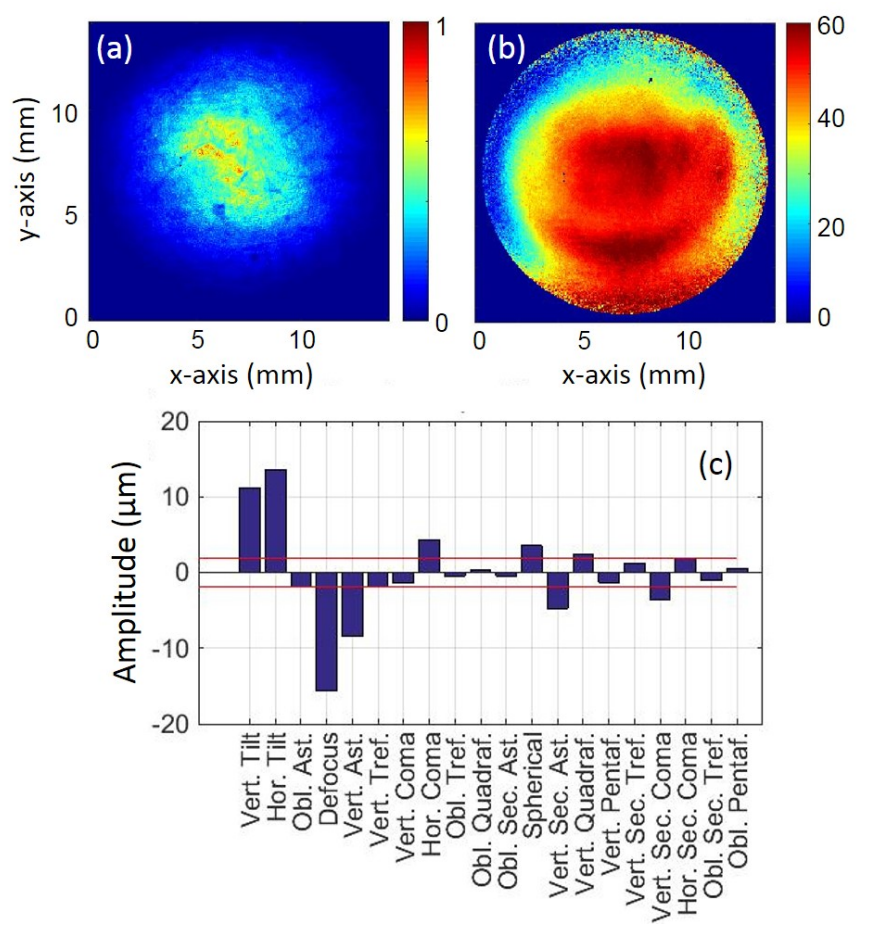

Fig. 2. Analysis of the reference beam at $0.75 \mathrm{THz}$. Amplitude (a) and wavefront (b) in the ZnTe crystal plane. (c) Amplitude of the Zernike coefficients. The horizontal red lines indicate the noise level.

To illustrate this measurement procedure, the shape of the DM is first simply set as a planar mirror. This situation will provide the measurements of both the amplitude and phase of the so-called "reference" $\mathrm{THz}$ wave for any frequencies within the 0-3 THz spectral range. Note that we rely here on the ability of our sensor to characterize broadband $\mathrm{THz}$ pulses. For instance, Fig. 2(a) represents the amplitude distribution of this reference THz wave at $\Omega=0.75 \mathrm{THz}$, at the crystal position. There, the beam diameter is $13.5 \mathrm{~mm}$, limited by the aperture of the DM which represents also the entrance pupil of the optical system. As shown in the figure, this entrance pupil is correctly imaged by the CMOS camera thanks to the $4 f^{\prime}$ optical configuration. The spatial inhomogeneity of the THz amplitude is due to the incoming pump laser beam and the optical rectification process in the first ZnTe crystal. We believe that the correction of this inhomogeneity could be addressed in further works by controlling the wavefront of the $\mathrm{THz}$ wave. To this aim, it would be necessary to modify the optical arrangement so that the phase shift induced by the DM would also change the amplitude of the $\mathrm{THz}$ wave in the crystal. Fig. 2(b) shows the $\mathrm{THz}$ wavefront at $\Omega=0.75 \mathrm{THz}$, at the crystal position. We note that it is not planar with both horizontal and vertical geometrical aberrations, in the order of tens of $\mu \mathrm{m} \mathrm{PV}$, from one side to the opposite side 
of the beam.

To quantify these aberrations, the standard method consists in developing the wavefront surface $W(x, y)$ onto a linear combination of the Zernike polynomial modes [16], $W(x, y)=$ $\sum_{i} a_{i} Z_{i}(x, y)$, where $a_{i}$ represents the amplitudes of the polynomial $Z_{i}$, each polynomial corresponding to one specific optical aberration. Fig. 2(c) represents the amplitudes in $\mu \mathrm{m}$ of the 20 first Zernike polynomial modes, after decomposition of the wavefront presented in Fig. 2(b). Except the vertical and horizontal tilts (tip/tilt) which are not intrinsic to the $\mathrm{THz}$ wave but mainly related with the overlapping with the laser probe beam, the main aberrations are negative defocus $(-16 \mu \mathrm{m})$, vertical astigmatism $(-8 \mu \mathrm{m})$, horizontal coma $(4 \mu \mathrm{m})$ and vertical secondary astigmatism $(5 \mu \mathrm{m})$. The other aberrations are smaller or close to the measurement limit accuracy $( \pm 2.5 \mu \mathrm{m})$, indicated by the horizontal red lines in the figure [14]. This noise level is as small as $\lambda / 160$ at $0.75 \mathrm{THz}$, emphasizing the high sensitivity of our wavefront sensor. From this first experiment, we can conclude that the reference $\mathrm{THz}$ wavefront is not perfectly planar with residual geometrical aberrations in the order of $\lambda / 40$ in terms of Zernike amplitudes.

Before correcting the reference $\mathrm{THz}$ wavefront, we have to verify that our wavefront sensor is able to measure the modifications induced by the DM on the optical system. For that, with the DM controller, we generated the wavefronts of 9 specific Zernike polynomials modes, such as horizontal tilt, defocus, vertical astigmatism, vertical trefoil, horizontal coma, oblique quadrafoil, oblique secondary astigmatism, spherical aberration and vertical secondary coma. For each mode, we set the amplitude of the Zernike coefficient $a_{i}$ as indicated in the second column in Table 1. Fig. 3(a)-(i) shows the wavefronts measured at $0.75 \mathrm{THz}$ corresponding to these 9 modes. These images have been obtained by subtracting the reference wavefront (Fig. 2(b)) in order to remove the intrinsic aberrations induced by the reference $\mathrm{THz}$ beam itself. Clearly, all Zernike modes are properly transferred to the $\mathrm{THz}$ wave and measured by the $\mathrm{THz}$ wavefront sensor. The third column in Table 1 gives the amplitudes of each mode, measured after developing each $\mathrm{THz}$ wavefront with respect to its corresponding mode. Considering that the measurement uncertainty is $\pm 2.5 \mu \mathrm{m}$ ), the experimental amplitudes are in good agreement with the amplitudes set by the DM controller, indicating the ability of our optical system to properly control an incoming $\mathrm{THz}$ wavefront.

Table 1. Amplitude of the Zernike modes measured by the THz sensor.

\begin{tabular}{cccc}
\hline Mode name & \multicolumn{3}{c}{ Set $(\mu \mathrm{m})$ Measured $(\mu \mathrm{m})$ Rel. error $(\%)$} \\
\hline Hor. tilt & 25 & 24.8 & 0.8 \\
Defocus & -20 & -18.3 & 8.5 \\
Vert. astigm. & -15 & 13.7 & 8.7 \\
Vert. trefoil & 7.5 & 7.1 & 5.3 \\
Hor. coma & 7.5 & 5.9 & 21.3 \\
Obl. quadrafoil & -7.5 & -7.5 & 0.0 \\
Obl. second. astigm. & -7.5 & -6.1 & 18.7 \\
Sph. aberration & -7.5 & -6.0 & 20.0 \\
Vert. second. coma & 7.5 & 5.2 & 30.7 \\
\hline
\end{tabular}

The final experiment consists in correcting the reference $\mathrm{THz}$ wavefront with the DM in order to remove the aberrations and optimize the optical configuration for further applications such

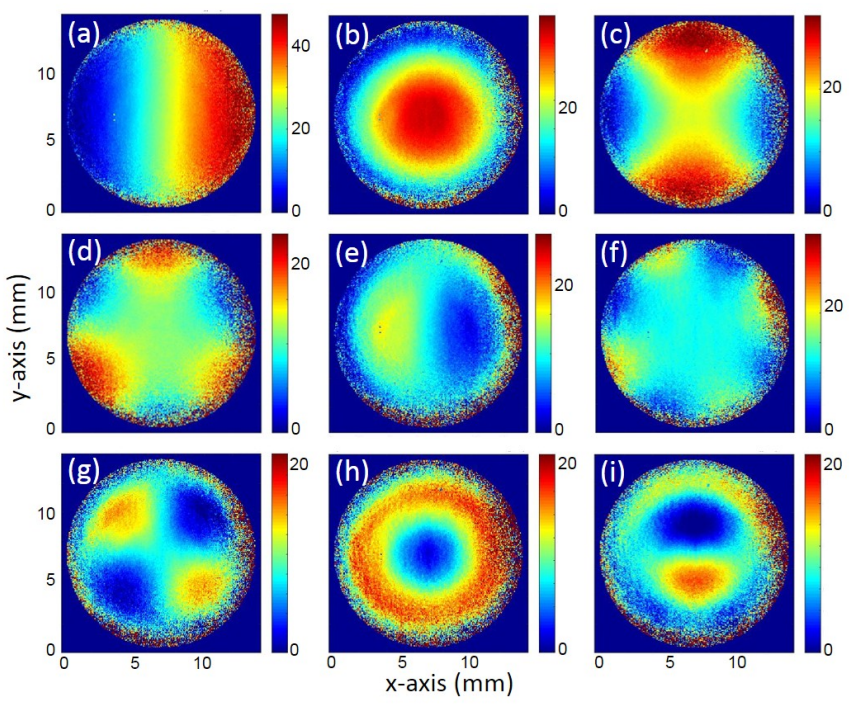

Fig. 3. Wavefronts at $0.75 \mathrm{THz}$ for different deformations of the DM. (a) Horizontal tilt. (b) Defocus. (c) Vertical astigmatism. (d) Vertical trefoil. (e) Horizontal coma. (f) Oblique quadrafoil. (g) Oblique secondary astigmatism. (h) Spherical aberration. (i) Vertical secondary coma.

as spectroscopy or imaging. First, as already shown in Fig. 2(b) and (c), we measure and develop the reference $\mathrm{THz}$ wavefront onto the first 20 Zernike polynomial modes $Z_{i}$. We thus obtain a set of 20 Zernike polynomial amplitudes $a_{i}$. Then, we program the DM controller so that the shape of the DM corresponds to $-2 \cos \alpha \times \sum_{i} a_{i} Z_{i}(x, y)$, where $\alpha=16^{\circ}$ is the incidence angle of the THz beam onto the DM. With this simple procedure, the specific shape of the DM will exactly compensate the $\mathrm{THz}$ wavefront after the reflection so that the geometrical aberrations will be remove in the $\mathrm{ZnTe}$ crystal since this position is conjugated with the DM thanks to the $4 f^{\prime}$ optical configuration of the experiment. Fig. 4 shows the result of this correction, at $0.75 \mathrm{THz}$, with the reference (a) and corrected (b) wavefronts, the reference (c) and the corrected (d) Zernike decompositions. Even if remaining aberrations are still observed, the amplitudes are all smaller than $5 \mu \mathrm{m}$ and close to the noise level of the wavefront sensor. We believe that the remaining aberrations are due to the limited courses of the DM actuators. Indeed, as explained before, this DM is adapted to visible and infrared light with a local deformation between two adjacent actuators of only $5 \mu \mathrm{m}$, which limits the maximum deformation of the whole mirror. For the correction presented in Fig. 2(b) and (c), the reference deformation in the center was out of range for a few actuators in this region, demonstrating that the DM is not perfectly adapted to our system. Nevertheless, this result demonstrates the ability of a DM to strongly attenuate the geometrical aberrations of a $\mathrm{THz}$ wave.

To evaluate the optimization of the THz wavefront, we calculated the Strehl ratio defined as the ratio of the on-axis intensities, in the presence and in the absence of aberrations. This number gives a measure of the quality of optical image formation [17]. It takes a value between 0 and 1, the latter corresponding to a perfectly unaberrated optical system. More precisely, the Strehl ratio can be expressed as $S=\exp \left[-(2 \pi \sigma)^{2}\right]$, where $\sigma$ is the RMS wavefront error in units of wavelength, directly related to the wavefront $W(x, y)$, by $\sigma^{2}=\left\langle W(x, y)^{2}\right\rangle-\langle W(x, y)\rangle^{2}$, where $\langle$. 

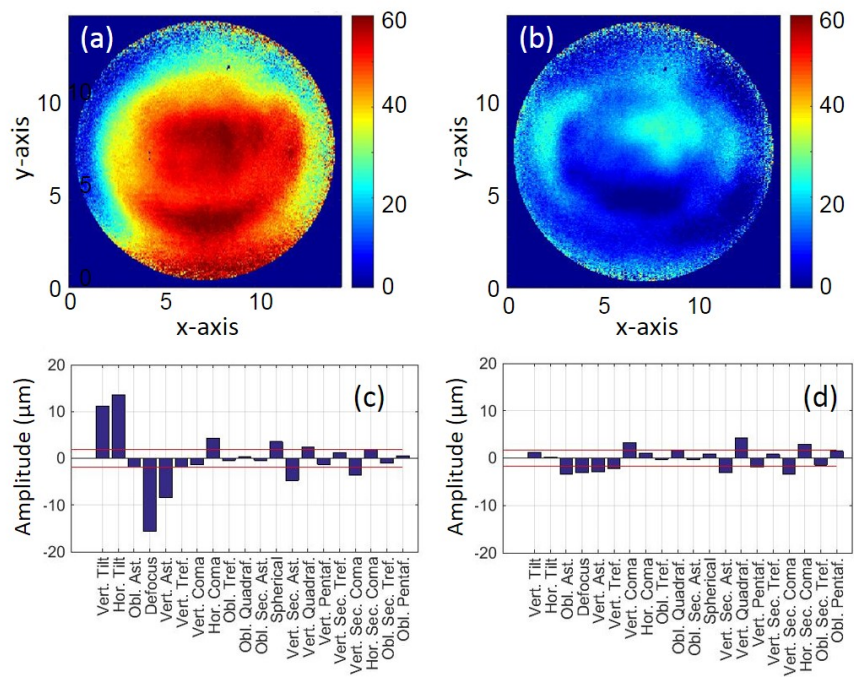

Fig. 4. Correction of the reference $\mathrm{THz}$ beam at $0.75 \mathrm{THz}$. (a) Reference wavefront. (b) Corrected wavefront. (c) Amplitude of the Zernike coefficients for (a). (d) Amplitude of the Zernike coefficients for (b). The horizontal red lines indicate the noise level.

is a spatial averaging. At $0.75 \mathrm{THz}$, we found $S=0.93$ for the reference wavefront and $S=0.97$ for the corrected one. This correction shows that the phase aberrations, already quite small prior to compensation since a Strehl ratio of $93 \%$ already corresponds to a diffraction-limited regime, are nearly fully corrected by the AO system. Using the same parameters for the DM deformation, i.e. adapted to the correction of the $0.75 \mathrm{THz}$ wavefront, it is important to evaluate if this correction is also effective for the other frequencies of the broadband $\mathrm{THz}$ wave. Therefore, we measured the reference and corrected $\mathrm{THz}$ wavefronts for other frequencies and calculated the corresponding Strehl ratios, being in mind that the DM shape is actually set for the correction of the $0.75 \mathrm{THz}$ wavefront. Fig. 5 represents the evolution of the reference and corrected Strehl ratios as a function of the frequency. We observe that the correction is effective in the 0.6$0.9 \mathrm{THz}$ spectral region. Out of this band, the corrected Strehl ratios become lower than the reference ones, indicating that the aberrations of the $\mathrm{THz}$ beam depend on the wavelength like chromatic aberrations.

In conclusion, by means of 2D EO imaging of $\mathrm{THz}$ waveforms and subsequent utilization of a DM, we were able to control and minimize the geometrical aberrations of a $\mathrm{THz}$ wave. The precision of the wavefront measurement is $\lambda / 60$, without any prior reference measurement. This work demonstrates the potential of $\mathrm{AO}$ technology for the optimization of $\mathrm{THz}$ beam quality. Further developments will consist in modifying the optical arrangement so that the phase shift induced by the DM will also provide the amplitude correction of the $\mathrm{THz}$ wave that will be essential for further practical or fundamental applications.

Acknowledgment. We thank ALPAO for the loan of the deformable mirror. We also thank the CNRS for specific financial support within the Interdisciplinary Mission, Instrumentation at the limits. The Conseil Régional Nouvelle Aquitaine and FEDER are also thanked for funding the equipments of the COLA platform at LOMA.

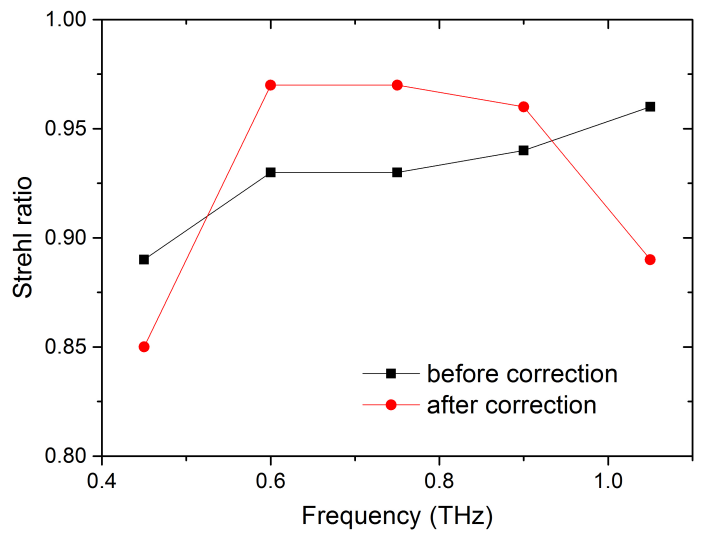

Fig. 5. Spectral dependence of the Strehl ratios for reference (before correction) and corrected wavefronts.

\section{us4pt inus3pt! REFERENCES}

1. P. Kern, P. Lena, P. Gigan, J.-C. Fontanella, G. Rousset, F. Merkle, and J.-P. Gaffard, Proc. SPIE 1130, 1130 (1989).

2. E. Gofas Salas, K. Grieve, S. Zwillinger, P. Mecê, C. Petit, J. Montri, J. Sahel, M. Paques, and S. Meimon 17, 42 (2017).

3. R. V. Shack and B. C. Platt, J. Opt. Soc. Am. 61, 656 (1971).

4. J.-F. Sauvage, T. Fusco, C. Petit, A. Costille, D. Mouillet, J.-L. Beuzit, K. Dohlen, M. Kasper, M. Suarez, C. Soenke, A. Baruffolo, B. Salasnich, S. Rochat, E. Fedrigo, P. Baudoz, E. Hugot, A. Sevin, D. Perret, F. Wildi, M. Downing, P. Feautrier, P. Puget, A. Vigan, J. O'Neal, J. Girard, D. Mawet, H. M. Schmid, and R. Roelfsema, Journal of Astronomical Telescopes, Instruments, and Systems 2, 025003 (2016).

5. W. L. Chan, J. Deibel, and D. M. Mittleman, Rep. Prog. Phys. 70, 1325 (2007).

6. N. J. Laurita, B. Cheng, R. Barkhouser, V. A. Neumann, and N. P. Armitage, Journal of Infrared, Millimeter, and Terahertz Waves 37, 894 (2016).

7. A. Bitzer, H. Helm, and M. Walther, IEEE J. Sel. Top. Quantum Electron. 14, 476 (2008).

8. M. Cui, J. N. Hovenier, Y. Ren, A. Polo, and J. R. Gao, Opt. Express 20, 14380 (2012).

9. M. Cui, J. N. Hovenier, Y. Ren, N. Vercruyssen, J. R. Gao, T. Y. Kao, Q. Hu, and J. L. Reno, Appl. Phys. Lett. 102, 111113 (2013).

10. H. Richter, M. Greiner-Bär, N. Deßmann, J. Pfund, M. Wienold, L. Schrottke, R. Hey, H. T. Grahn, and H.-W. Hübers, Appl. Phys. Lett. 101, 031103 (2012).

11. H. Richter, N. Rothbart, and H.-W. Hübers, Journal of Infrared, Millimeter, and Terahertz Waves 35, 686 (2014).

12. J. F. Molloy, M. Naftaly, and R. A. Dudley, IEEE J. Sel. Top. Quantum Electron. 19, 8401508 (2013).

13. E. Abraham, H. Cahyadi, M. Brossard, J. Degert, E. Freysz, and T. Yasui, Opt. Express 24, 5203 (2016).

14. M. Brossard, H. Cahyadi, M. Perrin, J. Degert, E. Freysz, T. Yasui, and E. Abraham, IEEE Transactions on Terahertz Science and Technology 7, 741 (2017).

15. Z. Jiang, X. G. Xu, and X.-C. Zhang, Appl. Opt. 39, 2982 (2000).

16. W. Southwell, J. Opt. Soc. Am. 70, 998 (1980).

17. K. Strehl, Zeitschrift für Instrumentenkunde pp. 213-217 (1902). 


\section{us4pt inus3pt! FULL REFERENCES}

1. P. Kern, P. Lena, P. Gigan, J.-C. Fontanella, G. Rousset, F. Merkle, and J.-P. Gaffard, "Come-on: An adaptive optics prototype dedicated to infrared astronomy," Proc. SPIE 1130, 1130 - 1130 - 12 (1989).

2. E. Gofas Salas, K. Grieve, S. Zwillinger, P. Mecê, C. Petit, J. Montri, J. Sahel, M. Paques, and S. Meimon, "Wide field $200 \mathrm{hz}$ videos of human retinas with paris's ao-fio," 17, 42 (2017).

3. R. V. Shack and B. C. Platt, "Production and use of a lenticular hartmann screen," J. Opt. Soc. Am. 61, 656-660 (1971).

4. J.-F. Sauvage, T. Fusco, C. Petit, A. Costille, D. Mouillet, J.-L. Beuzit, K. Dohlen, M. Kasper, M. Suarez, C. Soenke, A. Baruffolo, B. Salasnich, S. Rochat, E. Fedrigo, P. Baudoz, E. Hugot, A. Sevin, D. Perret, F. Wildi, M. Downing, P. Feautrier, P. Puget, A. Vigan, J. O'Neal, J. Girard, D. Mawet, H. M. Schmid, and R. Roelfsema, "SAXO: the extreme adaptive optics system of SPHERE (I) system overview and global laboratory performance," Journal of Astronomical Telescopes, Instruments, and Systems 2, 025003 (2016).

5. W. L. Chan, J. Deibel, and D. M. Mittleman, "Imaging with terahertz radiation," Rep. Prog. Phys. 70, 1325-1379 (2007).

6. N. J. Laurita, B. Cheng, R. Barkhouser, V. A. Neumann, and N. P. Armitage, "A Modified $8 f$ Geometry with Reduced Optical Aberrations for Improved Time Domain Terahertz Spectroscopy," Journal of Infrared, Millimeter, and Terahertz Waves 37, 894-902 (2016).

7. A. Bitzer, H. Helm, and M. Walther, "Beam-Profiling and WavefrontSensing of THz Pulses at the Focus of a Substrate-Lens," IEEE J. Sel. Top. Quantum Electron. 14, 476-481 (2008).

8. M. Cui, J. N. Hovenier, Y. Ren, A. Polo, and J. R. Gao, "Terahertz wavefronts measured using the Hartmann sensor principle," Opt. Express 20, 14380-14391 (2012).

9. M. Cui, J. N. Hovenier, Y. Ren, N. Vercruyssen, J. R. Gao, T. Y. Kao, Q. Hu, and J. L. Reno, "Beam and phase distributions of a terahertz quantum cascade wire laser," Appl. Phys. Lett. 102, 111113 (2013).

10. H. Richter, M. Greiner-Bär, N. Deßmann, J. Pfund, M. Wienold, L. Schrottke, R. Hey, H. T. Grahn, and H.-W. Hübers, "Terahertz wavefront measurement with a Hartmann sensor," Appl. Phys. Lett. 101, 031103 (2012).

11. H. Richter, N. Rothbart, and H.-W. Hübers, "Characterizing the beam properties of terahertz quantum-cascade lasers," Journal of Infrared, Millimeter, and Terahertz Waves 35, 686-698 (2014).

12. J. F. Molloy, M. Naftaly, and R. A. Dudley, "Characterization of Terahertz Beam Profile and Propagation," IEEE J. Sel. Top. Quantum Electron. 19, 8401508-8401508 (2013).

13. E. Abraham, H. Cahyadi, M. Brossard, J. Degert, E. Freysz, and T. Yasui, "Development of a wavefront sensor for terahertz pulses," Opt. Express 24, 5203 (2016).

14. M. Brossard, H. Cahyadi, M. Perrin, J. Degert, E. Freysz, T. Yasui, and E. Abraham, "Direct wavefront measurement of terahertz pulses using two-dimensional electro-optic imaging," IEEE Transactions on Terahertz Science and Technology 7, 741-746 (2017).

15. Z. Jiang, X. G. Xu, and X.-C. Zhang, "Improvement of terahertz imaging with a dynamic subtraction technique," Appl. Opt. 39, 2982-2987 (2000).

16. W. Southwell, "Wave-front estimation from wave-front slope measurements," J. Opt. Soc. Am. 70, 998-1006 (1980).

17. K. Strehl, "Über luftschlieren und zonenfehler," Zeitschrift für Instrumentenkunde pp. 213-217 (1902). 